\title{
3D FOCUSING IN PARALLEL MICROCHANNELS FOR HIGH-THROUGHPUT SYNTHESIS OF POLYMERIC NANOPARTICLES
}

\author{
Jong-Min Lim ${ }^{1,2,3}$, Pedro M. Valencia , Minsoung Rhee ${ }^{1,2,3}$, \\ Robert S. Langer ${ }^{3,4}$, Omid C. Farokhzad ${ }^{2},{ }^{*}$, and Rohit Karnik ${ }^{1,}{ }^{*}$
}

${ }^{1}$ Department of Mechanical Engineering, Massachusetts Institute of Technology, Cambridge, MA, USA

${ }^{2}$ Department of Anesthesiology, Brigham and Women's Hospital - Harvard Medical School, Boston, MA, USA

${ }^{3}$ Koch Institute for Integrative Cancer Research, Massachusetts Institute of Technology, Cambridge, MA, USA

${ }^{4}$ Department of Chemical Engineering, Massachusetts Institute of Technology, Cambridge, MA, USA

\begin{abstract}
We have developed a multilayer microfluidic device for the parallelization of nanoparticles synthesis by microfluidic rapid nanoprecipitation in 3D hydrodynamic flow focusing geometry. Since the shape and position of interconnecting holes between upper and lower poly(dimethylsiloxane) (PDMS) channels could be optimized, stable vertical focusing could be achieved. The poly(lactide-co-glycolide)- $b$-polyethyleneglycol (PLGA-PEG) nanoparticles with various sizes could be synthesized with polymers of different molecular weights and concentrations. In addition, the size of nanoparticles could be precisely tuned simply by changing the flow ratio of organic stream to the total flow rate of water when the molecular weights and concentrations of polymer were fixed.
\end{abstract}

\section{INTRODUCTION}

Use of microfluidics for synthesis of nanoparticles has been of great interest due to controllability in their physicochemical properties and continuous flow process ${ }^{1-3}$. Recently, our group demonstrated microfluidic synthesis of poly(lactide-co-glycolide)- $b$-polyethyleneglycol (PLGA-PEG) nanoparticles for drug delivery systems using rapid nanoprecipitation by mixing polymer solution in acetonitrile with water in hydrodynamic flow focusing geometry ${ }^{4}$. More recently, we reported a single channel microfluidic device with three sequential holes to achieve the 3D hydrodynamic flow focusing 5 . We could enhance the robustness of operation by adopting the $3 \mathrm{D}$ hydrodynamic flow focusing geometry that isolates the precipitating polymers from poly(dimethylsiloxane) (PDMS) channel walls, eliminating microchannel fouling. However, applications of the microfluidic synthesis were limited to in vitro studies due to low throughput. In addition, the precision of manual drilling was critical to achieve stable 3D hydrodynamic flow focusing.

Herein, we reported the parallelization of nanoparticles synthesis in microfluidic system by using multilayer microfluidic device that enable sufficient throughput for in-vivo studies. Since the interconnecting holes between upper and lower PDMS channels were originated from the patterned SU-8 posts, the shape and position of interconnecting holes could be optimized. In addition, the precision of the interconnecting holes position could be significantly enhanced by using conventional multilayer photolithography. As a result, more stable vertical focusing could be achieved. Moreover, only four external tubing connections were required due to the 3D fluidic architecture design.

Smaller nanoparticles could be synthesized reproducibly from the microfluidic rapid nanoprecipitation method since the mixing time scale was shorter than that of conventional bulk synthesis method. In addition, the size of nanoparticles could be precisely controlled simply by changing the flow rates of fluids when the molecular weights and concentrations of polymer were fixed. Because the threshold of renal clearance of nanoparticles is about $5.5 \mathrm{~nm}$ and the minimum size from conventional bulk synthesis method is $30 \mathrm{~nm}^{6,7}$, the sub-30 nm PLGA-PEG nanoparticles synthesized from 3D hydrodynamic flow focusing can be used for optimizing the size of nanomedicine ${ }^{8}$.

\section{EXPERIMENTAL}

\section{Materials}

As the polymeric precursor, PLGA-PEG (Boehringer Ingelheim $\mathrm{GmbH}$, Germany) at molecular weights of $\mathrm{PLGA}_{10 \mathrm{~K}}-\mathrm{PEG}_{5 \mathrm{~K}}$, $\mathrm{PLGA}_{27 \mathrm{~K}}-\mathrm{PEG}_{5 \mathrm{~K}}, \mathrm{PLGA}_{45 \mathrm{~K}}-\mathrm{PEG}_{5 \mathrm{~K}}$, and $\mathrm{PLGA}_{95 \mathrm{~K}}-\mathrm{PEG}_{5 \mathrm{~K}}$ were dissolved in acetonitrile (Aldrich) at concentrations of $10-50$ $\mathrm{mg} / \mathrm{mL}$. Pure acetonitrile and deionized water were employed as vertical and lateral sheath stream, respectively. To obtain the confocal microscope images, aqueous solution of Rh6G (Aldrich) and FITC (Aldrich) were used as core and vertical sheath fluids, respectively.

\section{Preparation of Multilayer PDMS Microfluidic device}

Multilayer 3D hydrodynamic flow focusing microfluidic device was prepared using multilayer photolithography and multilayer soft lithography 9 . The multilayer device preparation procedure was illustrated in Figure 1. Master molds for the bottom and upper layers were patterned in SU-8 photoresist via conventional photolithography. Post arrays for interconnecting holes were patterned in SU-8 on top of the bottom layer with precise alignment using an UV aligner.

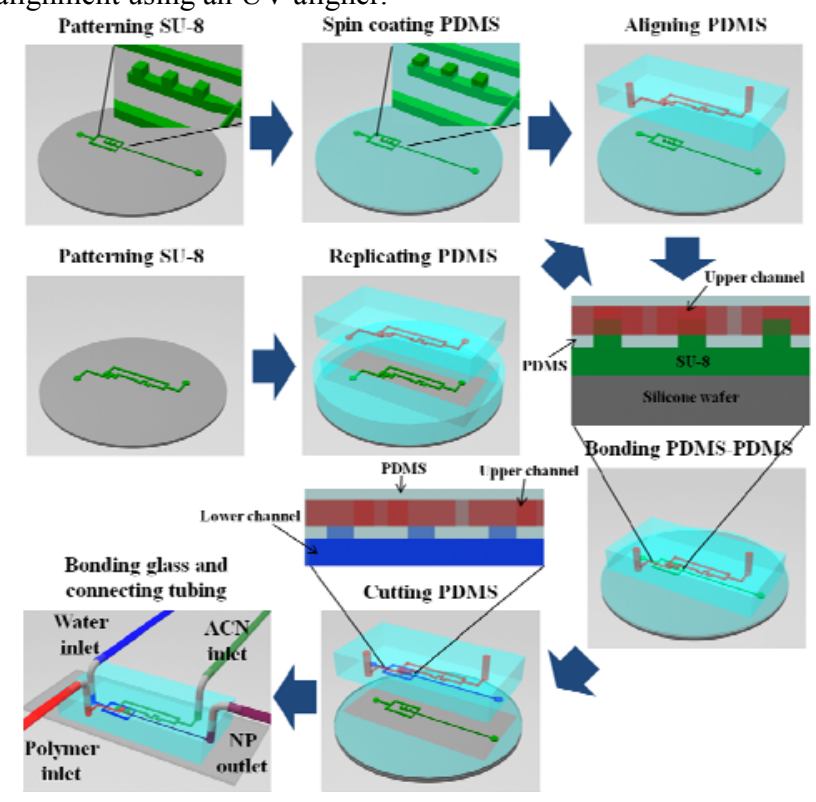

Figure 1: Schematic illustration of fabrication procedure for the multilayer $3 D$ hydrodynamic flow focusing device. 
A thin PDMS membrane was spin-coated on the master mold for bottom layer with post arrays. The spin speed was tuned to precisely control the membrane thickness to be slightly lower than the interconnect post height. The upper PDMS microchannel was prepared using conventional replica molding of PDMS from the SU-8 master for upper layer. The upper PDMS channel was aligned under a microscope and bonded on top of bottom PDMS membrane using oxygen plasma treatment. To assemble the final device, the multilayer PDMS channel was bonded with a slide glass via oxygen plasma and connected to tubing.

\section{Preparation of PLGA-PEG Nanoparticles}

Monodisperse PLGA-PEG nanoparticles were synthesized using the multilyer PDMS microfluidic device. The PLGA-PEG precursor in acetonitrile was introduced to the microchannel connected with the middle interconnecting holes. Pure acetonitrile was introduced to the microchannel connected with the first and the third interconnecting holes. The water flow rate were maintained at $50 \mu \mathrm{L} / \mathrm{min}$ and $400 \mu \mathrm{L} / \mathrm{min}$ for single $3 \mathrm{D}$ hydrodynamic flow focusing and 8 parallel 3D hydrodynamic flow focusing, respectively. During the nanoparticle synthesis, the flow rates were controlled by syringe pumps (Harvard Apparatus) and the flow motion was observed using an inverted microscope (Nikon, Eclipse TS 100). To confirm the vertical focusing, laser scanning confocal microspcope (Carl Zeiss MicroImaging, LSM 510) was used. In case of the bulk synthesis, we mixed $100 \mu \mathrm{L}$ of polymeric precursor solution drop-wise with $1 \mathrm{~mL}$ of water for about $2 \mathrm{hrs}^{10,11}$.

\section{PLGA-PEG Nanoparticles Characterization}

The size distribution by volume fraction of PLGA-PEG nanoparticles were measured using dynamic light scattering with Zetasizer Nano ZS (Malvern Instruments Ltd., U.K.). It is noteworthy that the nanoparticles size did not vary significantly after washing with deionized water several times. The synthesized PLGA-PEG nanoparticles were imaged by TEM (JEOL 200CX) with negative staining by uranyl acetate (Electron Microscopy Sciences).

\section{RESULTS AND DISCUSSION}

Multilayer PDMS microfluidic device for parallel 3D hydrodynamic flow focusing could be prepared using multilayer photolithography and multilayer soft lithography ${ }^{9}$. Figure 2 shows the digital camera image of the 8 parallel 3D hydrodynamic flow focusing device. To clearly demonstrate the upper and lower microchannels, the interconnecting holes were closed by spincoating the PDMS thicker than the height of post arrays. In addition, the red and blue food coloring dyes were filled in the upper and lower microchannels, respectively.

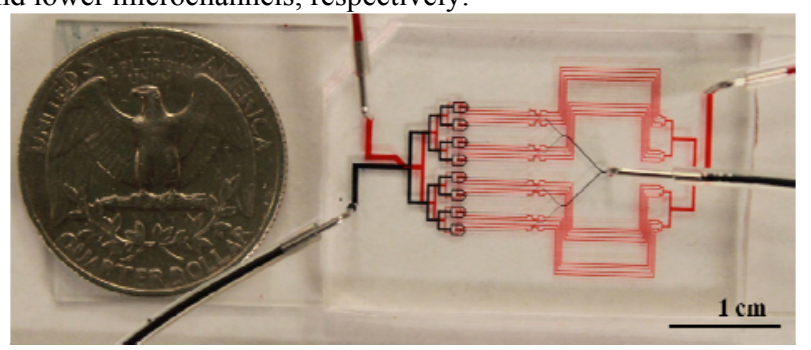

Figure 2: Digital camera images of 8 parallel 3D hydrodynamic flow focusing device.

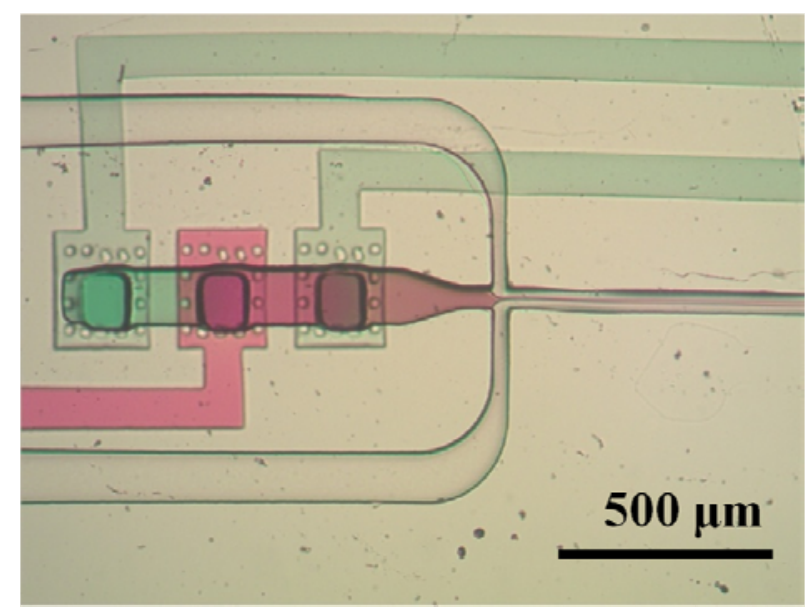

Figure 3: Optical microscope image of the 3D hydrodynamic flow focusing device.

It is noteworthy that the microchannels for the upper sheath stream were meandered to match the resistances of the microchannel lengths between upper and bottom streams.

Figure 3 shows optical microscope image of the device for nanoparticles synthesis. In case of the device used for nanoparticle synthesis, the thickness of spincoated PDMS was slightly lower than the height of post arrays (see Figure 1). As a result, the organic stream could flow from the upper microchannel to the lower microchannel. To confirm the interlayer flow, polymeric precursor and pure acetonitrile were denoted using red and green food coloring dyes, respectively (see Figure 3). Since the interlayer flows occurred only at the interconnecting holes, tubing of the device could be significantly simplified by using the multilayer microfluidic device. As a result, only 3 inlets and 1 outlet are required for a parallel 3D hydrodynamic flow focusing device.

The lateral position of inlet holes significantly affects the vertical focusing in 3D hydrodynamic flow focusing with three sequential inlets. The precision of manual drilling was critical in our previous single layer $3 \mathrm{D}$ hydrodynamic flow focusing device ${ }^{5}$. In case of the multilayer 3D hydrodynamic flow focusing device, precise alignment of interconnecting holes could be achieved by using conventional multilayer photolithography with UV aligner. In addition, the shape of interconnecting holes could be optimized. The effect of interconnecting holes geometry could be examined by using 3D finite element simulations using COMSOL (COMSOL Inc., Burlington, MA). Because deep microchannel with inlet holes having slightly larger diameter than the width of microchannel could achieved the most uniform distribution of vertically focused stream $^{5}$, the width and height of lower microchannel were fixed to $100 \mu \mathrm{m}$ and the width of interconnecting holes were fixed to $120 \mu \mathrm{m}$ during the 3D finite element simulations. Out of three sequential interconnecting holes, the first interconnecting hole for the bottom sheath fluid was excluded during the simulation because its influence to the vertical focusing is negligible. Round interconnecting holes resulted in the non-uniform distribution of concentration profile (see Figure 4a). Likewise, rectangular interconnecting holes smaller than $100 \mu \mathrm{m}$ in the lengthwise direction resulted in arched vertically focused stream (see Figure $4 \mathrm{~b}$ ). When rectangular interconnecting holes with larger than 100 $\mu \mathrm{m}$ in the lengthwise direction were used, the most uniform concentration profile of the vertically focused stream could be achieved (see Figure 4c and 4d). 


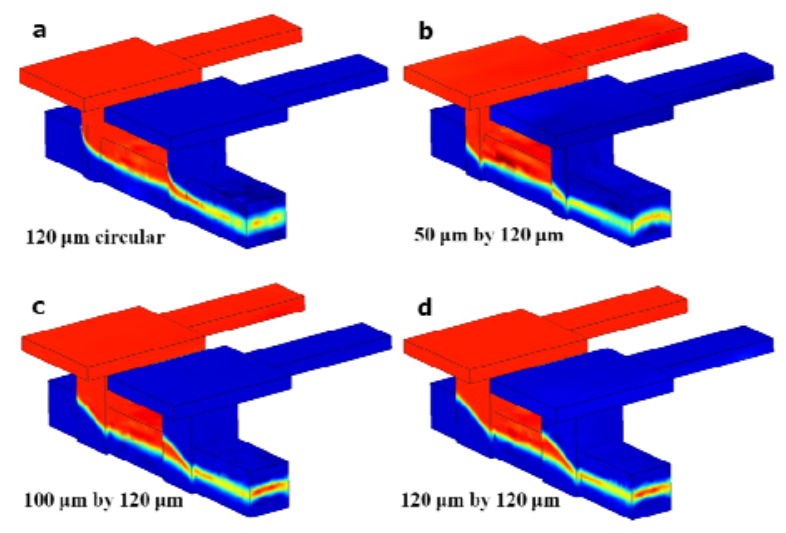

Figure 4: Perspective views of $3 D$ finite element simulations at the vertical focusing region with (a) round (120 $\mu \mathrm{m}$ in diameter), (b) rectangular $(50 \mu \mathrm{m} \times 120 \mu \mathrm{m})$, (c) rectangular $(100 \mu \mathrm{m} \times 120 \mu \mathrm{m})$, and $(d)$ square $(120 \mu \mathrm{m} \times 120 \mu \mathrm{m})$ shapes of interconnecting holes. Here, the fraction of polymer flow in organic flow $(f)$ is 0.3 . Both width and height of lower microchannel are $100 \mu \mathrm{m}$.

Since some residual PDMS may remain on top of the post arrays during the spincoating of PDMS if the length of post arrays were too long, the length of interconnecting holes was fixed at $100 \mu \mathrm{m}$ for the device fabrication.

The flow within the multilayer 3D hydrodynamic flow focusing device was examined using confocal microscopy. We used aqueous Rh6G (red) and FITC (green) fluorescent dye solutions as the vertically focused stream and the vertical sheath streams, respectively. As predicted in 3D finite element simulations, stable and uniform vertical focusing could be achieved when the rectangular interconnecting holes $(100 \mu \mathrm{m} \times 120 \mu \mathrm{m})$ were used (see Figure 5a and 5b).
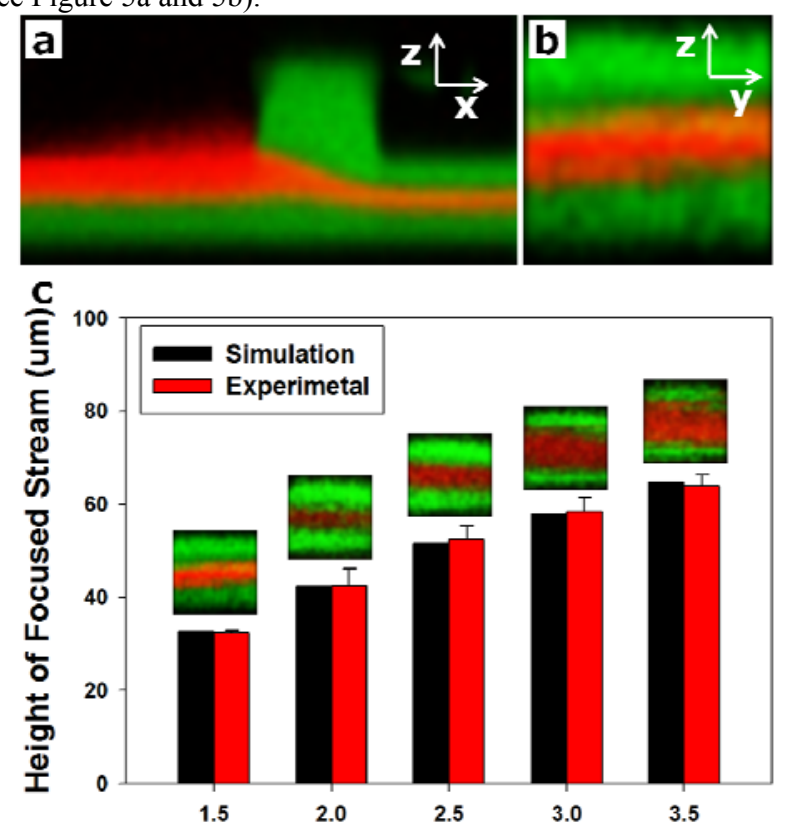

Fraction of Polymer Flow in Organic Flow

Figure 5: (a) cross-sectional front view and (b) cross-sectional side view from confocal microscope data at the vertical focusing region (c) Height of vertically focused polymeric stream as a function of the fraction of polymer flow in organic flow $(f)$.
In addition, the heights and shapes of vertical focusing streams obtained by confocal microscopy are collectively in good agreement with the 3D finite element simulations at various fraction of polymer flow in organic flow $(f)$ (see Figure $5 \mathrm{c}$ ). Since the resistances of microchannels for the vertical sheath streams were matched by meandering the microchannel for the upper sheath stream, the core stream could be located at the middle of the bottom microchannel. In addition, uniform concentration profile of the vertically focused stream could be achieved due to the optimal shapes of interconnecting holes. As a result, the vertically focused stream did not touch the PDMS microchannels even at high $f$ (see Figure 5c).

To enhance the production yield of nanoparticles, we performed parallelization of nanoparticles synthesis by using the multilayer microfluidic 3D hydrodynamic flow focusing. By using the multilayer PDMS microfluidic device, PLGA-PEG nanoparticles with various sizes could be synthesized with polymers of different molecular weights and concentrations (see Figure 6a). The nanoparticles synthesized from the microfluidic 3D hydrodynamic flow focusing were smaller compared to the nanoparticles synthesized from the bulk synthesis method because the mixing time $\left(\tau_{\text {mix }}\right)$ is shorter than the characteristic aggregation time scale $\left(\tau_{\text {agg }}\right)$ in the microfluidic rapid mixing ${ }^{12}$.
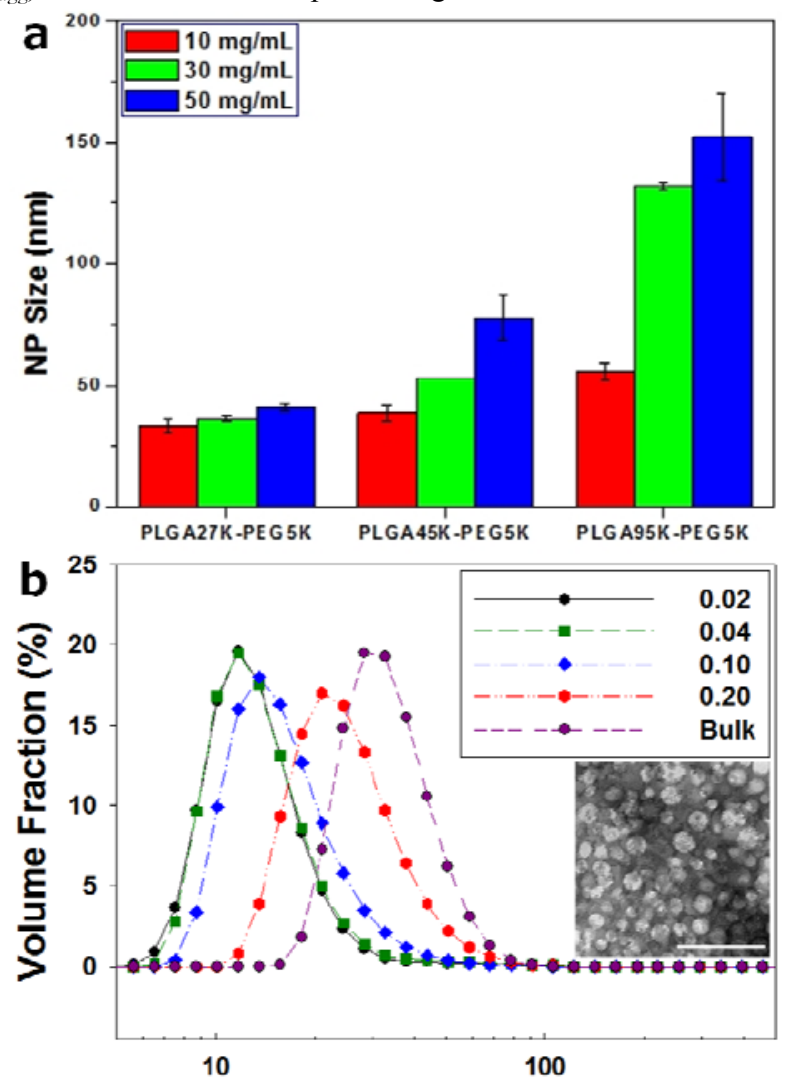

Nanoparticle Diameter (nm)

Figure 6: (a) Effect of PLGA-PEG precursor concentrations and molecular weights on the size of synthesized PLGA-PEG nanoparticles. (b) Effect of flow ratio on nanoparticles size. The inset shows TEM image of the nanoparticles prepared by microfluidic $3 D$ hydrodynamic flow focusing when the flow ratio of organic stream to the total flow rate of water $(R)$ is 0.04 . Scale bar is $100 \mathrm{~nm}$. 
When molecular weight and concentration of polymeric precursor are same, the size of nanoparticles depends on the mixing time $\left(\tau_{\text {mix }}\right)$ for hydrodynamic flow focusing ${ }^{4}$. The mixing time $\left(\tau_{\text {mix }}\right)$ for hydrodynamic flow focusing can be estimated as follows:

$$
\tau_{\text {mix }} \sim \frac{w_{f}^{2}}{4 D} \approx \frac{w^{2}}{9 D} \frac{1}{(1+1 / R)^{2}}
$$

Where $D$ is diffusivity of the solvent, $w_{f}$ is width of the focused stream, $w$ is channel width, and $R$ is the ratio of flow rate of the polymeric stream to the total flow rate of water. Since $\tau_{\text {mix }}$ for hydrodynamic flow focusing is function of flow ratio of organic stream to the total flow rate of water $(R)$, the size of nanoparticles could be controlled in 3D hydrodynamic flow focusing simply by changing the flow ratio of organic stream to the total flow rate of water $(R)$. Eq. 1 predicts mixing time $\left(\tau_{\operatorname{mix}}\right)$ in the range of 0.017 $1.2 \mathrm{~ms}$ for typical flow ratios $(\mathrm{R}=0.02-0.2)$ in our device. The size of nanoparticles decreased from about $24 \mathrm{~nm}$ to $14 \mathrm{~nm}$ as the mixing time $\left(\tau_{\text {mix }}\right)$ decreased when $\mathrm{PLGA}_{10 \mathrm{~K}}-\mathrm{PEG}_{5 \mathrm{~K}}$ with low concentration (i.e. $10 \mathrm{mg} / \mathrm{mL}$ ) was used as the polymeric precursor (see Figure $6 \mathrm{~b}$ ). It is noteworthy that the decrease in the nanoparticles size saturated when mixing time $\left(\tau_{\text {mix }}\right)$ was shorter than $0.066 \mathrm{~ms}$ (i.e. $\mathrm{R}=0.04$ ).

\section{CONCLUSION}

We could demonstrate the parallelization of PLGA-PEG nanoparticle synthesis in a microfluidic system by using multilayer microfluidic device. Since the shape and position of interconnecting holes between upper and lower PDMS channels could be optimized, stable vertical focusing could be achieved even at high fraction of polymer flow in organic flow $(f)$. The PLGA-PEG nanoparticles with various sizes could be synthesized with polymers of different molecular weights and concentrations. The nanoparticles synthesized from the microfluidic 3D hydrodynamic flow focusing were smaller compared to the nanoparticles synthesized from the bulk synthesis method because the mixing time $\left(\tau_{\text {mix }}\right)$ is shorter than the characteristic aggregation time scale $\left(\tau_{\text {agg }}\right)$ in the microfluidic rapid mixing. In addition, the size of nanoparticles could be precisely tuned simply by changing the flow ratio of organic stream to the total flow rate of water $(R)$ when the molecular weights and concentrations of polymer were fixed.

\section{ACKNOWLEDGMENTS}

This research was supported by the Koch-Prostate Cancer Foundation Award in Nanotherapeutics (R.L. and O.C.F.), by the Concept Development Grant 5P50CA090381-09 from the Dana Farber Cancer Institute Prostate SPORE (O.C.F.), and by NIH Grants CA119349 (R.L. and O.C.F.) and EB003647 (O.C.F.). P.M.V. is supported by NSF Graduate Research Fellowship.

\section{REFERENCES}

[1] Y. J. Song, J. Hormes, and C. Kumar, "Microfluidic synthesis of nanomaterials," Small, 4, 698 (2008).

[2] A. Jahn, J. E. Reiner, W. N. Vreeland, D. L. DeVoe, L. E. Locascio, and M. Gaitan, "Preparation of nanoparticles by continuous-flow microfluidics," Journal of Nanoparticle Research, 10, 925 (2008).

[3] S. Marre and K. F. Jensen, "Synthesis of micro and nanostructures in microfluidic systems," Chemical Society Reviews, 39, 1183 (2010).

[4] R. Karnik, F. Gu, P. Basto, C. Cannizzaro, L. Dean, W. Kyei-Manu, R. Langer, and O. C. Farokhzad, "Microfluidic platform for controlled synthesis of polymeric nanoparticles," Nano Letters, 8, 2906 (2008).

[5] M. Rhee, P. M. Valencia, M. I. Rodriguez, R. Langer, O. C. Farokhzad, and R. Karnik, "Synthesis of Size-Tunable Polymeric Nanoparticles Enabled by 3D Hydrodynamic Flow Focusing in Single-Layer Microchannels," Advanced Materials, 23, H79 (2011).

[6] H. S. Choi, W. Liu, P. Misra, E. Tanaka, J. P. Zimmer, B. I. Ipe, M. G. Bawendi, and J. V. Frangioni, "Renal clearance of quantum dots," Nature Biotechnology, 25, 1165 (2007).

[7] F. Alexis, E. Pridgen, L. K. Molnar, and O. C. Farokhzad, "Factors affecting the clearance and biodistribution of polymeric nanoparticles," Molecular Pharmaceutics, 5, 505 (2008).

[8] H. Cabral, Y. Matsumoto, K. Mizuno, Q. Chen, M. Murakami, M. Kimura, Y. Terada, M. R. Kano, K. Miyazono, M. Uesaka, N. Nishiyama, and K. Kataoka, "Accumulation of sub-100 nm polymeric micelles in poorly permeable tumours depends on size," Nature Nanotechnology, 6, 815 (2011).

[9] E. P. Kartalov, C. Walker, C. R. Taylor, W. F. Anderson, and A. Scherer, "Microfluidic vias enable nested bioarrays and autoregulatory devices in Newtonian fluids," Proceedings of the National Academy of Sciences of the United States of America, 103, 12280 (2006).

[10] J. Cheng, B. A. Teply, I. Sherifi, J. Sung, G. Luther, F. X. Gu, E. Levy-Nissenbaum, A. F. Radovic-Moreno, R. Langer, and O. C. Farokhzad, "Formulation of functionalized PLGA-PEG nanoparticles for in vivo targeted drug delivery," Biomaterials, 28, 869 (2007).

[11] J. M. Chan, P. M. Valencia, L. F. Zhang, R. Langer, and O. C. Farokhzad, "Polymeric Nanoparticles for Drug Delivery," in Cancer Nanotechnology: Methods and Protocols. vol. 624, ed Totowa: Humana Press Inc, 2010, pp. 163-175.

[12] B. K. Johnson and R. K. Prud'homme, "Mechanism for rapid self-assembly of block copolymer nanoparticles," Physical Review Letters, 91, 118302 (2003).

\section{CONTACT}

* R. Karnik, tel: +1-617-324-1155; karnik@mit.edu

* O. C. Farokhzad, tel: +1-617-732-6093; ofarokhzad@partners.org 